\title{
Hydrogen gas sensor based upon perylene-imide derivatives
}

\section{Kazuyuki Sato}

Department of Applied Physics, Graduate School of Engineering, Yokohama National University,

79-5 Tokiwadai, Hodogaya-ku, Yokohama 240-8501, Japan

E-mail: d05gd224@ynu.ac.jp

\section{Kazuyuki Hino}

Department of Applied Physics, Graduate School of Engineering, Yokohama National University, Yokohama, Japan

E-mail: Hino-K@mail.dnp.co.jp

\section{Hiroo Takahashi}

Department of Applied Physics, Graduate School of Engineering, Yokohama National University, Yokohama, Japan

E-mail: takahashi@ynu.ac.jp

\section{Jin Mizuguchi}

Department of Applied Physics, Graduate School of Engineering, Yokohama National University, Yokohama, Japan

E-mail: mizu-j@ynu.ac.jp

\begin{abstract}
Perylene-imide derivatives are industrially important pigments that exhibit a variety of shades from red via maroon to black. In the present investigation, we have synthesized novel perylene-imide derivatives with pyridyl rings in an attempt to apply them for $\mathrm{H}_{2}$ gas sensors based upon proton acceptors. The two $\mathrm{N}$ atoms of the pyridyl rings which work as proton acceptors are found to remain unbonded in the solid state according to X-ray analysis and are thus available for protonation necessary for $\mathrm{H}_{2}$ gas sensors. The $\mathrm{H}_{2}$ gas sensors exhibit a significant change in electrical resistivity by about three orders of magnitude even under $0.01 \% \mathrm{H}_{2}$.
\end{abstract}

Keywords: perylene-imide; hydrogen gas sensor; crystal structure.

Reference to this paper should be made as follows: Sato, K., Hino, K., Takahashi, H. and Mizuguchi, J. (xxxx) 'Hydrogen gas sensor based upon perylene-imide derivatives', Int. J. Intelligent Systems Technologies and Applications, Vol. X, No. Y, pp.000-000.

Biographical notes: Kazuyuki Sato received his Bachelor of Engineering from Yokohama National University in 2005. He is currently in the graduate course for applied physics at the same university. His research interest includes structure analysis of organic pigments and their electronic applications. 


\begin{abstract}
Author
Kazuyuki Hino received his Bachelor of Engineering in 2003 and his Master of Engineering in 2005, both from Yokohama National University. Currently, he works at Dai Nippon Printing Co., Ltd.

Hiroo Takahashi received his Bachelor of Engineering from Waseda University in 1968 and Dr. of Engineering from Yokohama National University in 2005. He worked at Sony Corporation from 1968 to 2004. Since 2004, he has been working as a visiting scientist at Yokohama National University.

Jin Mizuguchi obtained his B. Sc. in chemistry from Sophia University in 1970, Dr. of Science from the University of Tokyo in 1982 and Venia Docendi from the University of Bern in 1994. He worked at Sony Corporation Research Centre from 1970 to 1985 and Ciba-Geigy AG (Switzerland) from 1985 to 1995. Since 1995, Prof. Mizuguchi has been at Yokohama National University as professor of materials science.
\end{abstract}

\title{
1 Introduction
}

Perylene-imide derivatives are industrially important organic pigments that exhibit a variety of shades from red via maroon to black (Herbst and Hunger, 1993). These materials have also attracted attention as photoconductors for electrophotographic photoreceptors (Loufty, 1989; Duff et al., 1990) as well as materials for optical disks (Mizuguchi, 1998). We report here a new application of perylene-imide pigments with two pyridyl rings (Fig. 1) for $\mathrm{H}_{2}$ gas sensors.

We have recently developed a novel $\mathrm{H}_{2}$ gas sensor utilizing a high proton affinity of 1,4-diketo-3,6-bis-(4'-pyridyl)-pyrrolo-[3,4-c]pyrrole ( $p$-DPPP: Fig. 2(a)) (Takahashi and Mizuguchi, 2005). $p$-DPPP is a dipyridyl derivative of 1,4-diketo-3,6-diphenyl-pyrrolo[3,4-c]pyrrole (DPP: Fig. 2(b)) used widely as a red pigment (Herbst and Hunger, 1993). $p$-DPPP exhibits a vivid red colour like DPP and is quite stable against heat and light irradiation. However, a drastic colour change occurs from red to violet when exposed to the vapour of nitric acid, accompanied by a reduction in electrical resistivity by five orders of magnitude (Mizuguchi et al., 1993). The present outstanding effect is found to occur due to protonation at the $\mathrm{N}$ atom of the pyridyl ring and indicates that $p$-DPPP can be used as a material for acid sensors. However, in view of the advent of fuel cells in the near future, we tried to transform it into $\mathrm{a}_{2}$ gas sensor with the following idea. We believed that $\mathrm{H}_{2}$ can dissociate into atomic hydrogens even in the solid state in the presence of Pd or Pt (which works as a hydrogen trap) assisted by a high electric field. Then, the atomic hydrogen $(\mathrm{H})$ protonates $p$-DPPP at the $\mathrm{N}$ atom of the pyridyl ring to release an electron that contributes to the electrical current: $\mathrm{H}+$ pyridyl ring $\rightarrow$ protonated pyridyl ring $\left(\mathrm{N}^{+} \mathrm{H}\right)+\mathrm{e}^{-}$. We have realized these functions in interdigital electrodes as shown in Fig. 3(a) where a trace of Pd or Pt is directly sputtered onto ITO (indium-tin-oxide) electrodes in the form of islands (ca. $3 \AA$ ) (Fig. 3(b)). Then, $p$-DPPP layer is applied by vacuum evaporation ( $c a .300 \AA$ ). $\mathrm{H}_{2}$ is first adsorbed on the surface of $p$-DPPP and diffuses into the bulk where $\mathrm{H}_{2}$ encounters sputtered Pd. Then, $\mathrm{H}_{2}$ dissociates into hydrogen atoms under a high electric field: $\mathrm{H}_{2} \rightarrow \mathrm{H}+\mathrm{H}$. At this moment, the $\mathrm{N}$ atom of the pyridyl ring of $p$-DPPP (strong proton acceptor) captures the proton by releasing an electron. The sensor exhibits a remarkable reduction of resistivity by two 
Title

orders of magnitude even under $0.05 \% \mathrm{H}_{2}$. It is also interesting to note that only paraDPPP is effective for $\mathrm{H}_{2}$ gas sensors while ortho and meta-isomers give poor sensitivity. This is because the $\mathrm{N}$ atom of the pyridyl ring is totally blocked due to the formation of $\mathrm{NH}^{\cdots} \cdot \mathrm{N}$ intermolecular hydrogen bonds between the $\mathrm{NH}$ group of one molecule and the $\mathrm{N}$ atom of the pyridyl ring of the neighbouring one (Imoda et al., 2005; Hirota et al., 2006).

In the present investigation, we extend our idea to a larger chromophore such as a perylene-imide skeleton. We have synthesized novel perylene-imide derivatives with pyridyl rings in an attempt to apply them for $\mathrm{H}_{2}$ gas sensors. There are ortho, meta and para-pyridyl derivatives abbreviated to OPP, MPP and PPP, respectively (Fig. 1). These compounds are found to exhibit a better sensitivity by one order of magnitude as compared with that of $p$-DPPP. Since the free state (i.e. unbonded state) of the $\mathrm{N}$ atom of the pyridyl ring is prerequisite for $\mathrm{H}_{2}$ gas sensors, special attention has been paid to the crystal structure of the perylene-imide derivatives.

Figure 1 Molecular conformation: (a) OPP, (b) MPP and (c) PPP

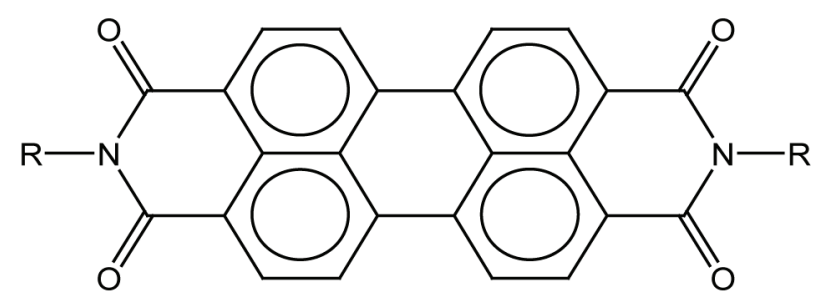

(a)

(b)

(c)

R:

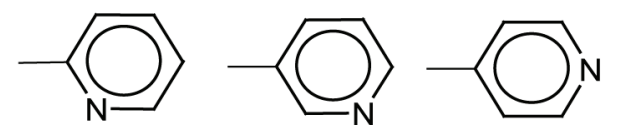

Figure 2 Molecular conformation: (a) $p$-DPPP and (b) DPP

(a)<smiles>O=C1NC(c2ccncc2)=C2C(=O)NC(c3ccncc3)=C12</smiles>

(b)<smiles>O=C1NC(c2ccccc2)=C2C(=O)NC(c3ccccc3)=C12</smiles> 
Author

\section{Experiment}

\subsection{Synthesis and crystal growth}

OPP was synthesized by heating perylene-3,4,9,10-tetracarboxylic dianhydride and 2aminopyridine at $490 \mathrm{~K}$ in dimethylnaphthalene for 3 hours. Likewise, MPP and PPP were synthesized with 3-aminopyridine and 4-aminopyridine, respectively. The products were then purified three times by sublimation at $760 \mathrm{~K}$, using a two-zone furnace (Mizuguchi, 1981). Single crystals of OPP and MPP were grown from the vapour phase. On the other hand, single crystals of PPP were grown by recrystallization from solution in 1-chloronaphthalene using an autoclave.

\subsection{Fabrication of $\mathrm{H}_{2}$ sensors based upon OPP, MPP and PPP}

The interdigital electrodes made of ITO (Fig. 3(a)) were prepared by lithographic techniques. Then Pd was sputtered directly onto the electrodes by a sputter equipment (E1030 Ion Sputter from Hitachi Corporation) in such a way as to form islands of Pd so as to avoid their contacts (Fig. 3(b)) (thickness: $c a$. $3 \AA$ ). After that, a thin layer of peryleneimide was applied by vacuum evaporation (Tokyo Vacuum Co., Ltd.: model EG240) to the thickness of about $300 \AA$.

Figure 3 (a) Interdigital electrodes and (b) magnified Pd-sputtered electrodes

(a)

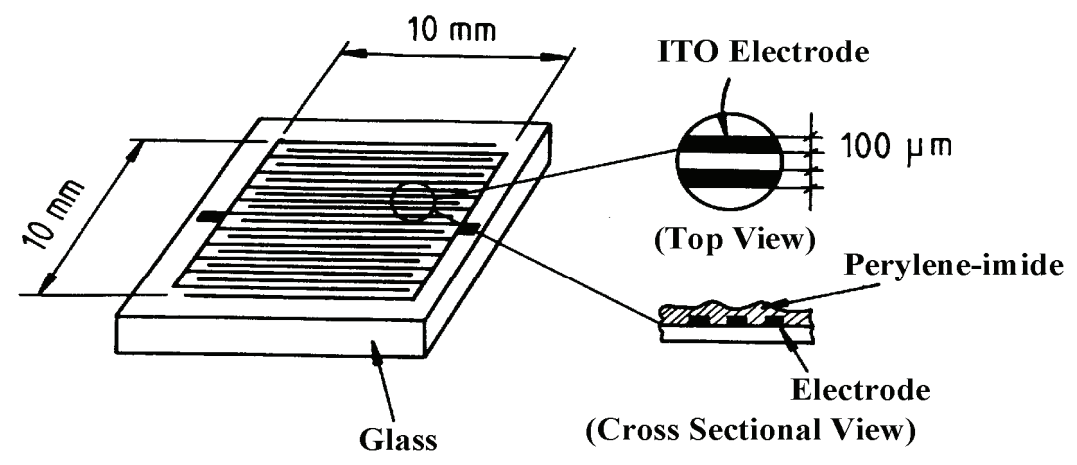

(b)

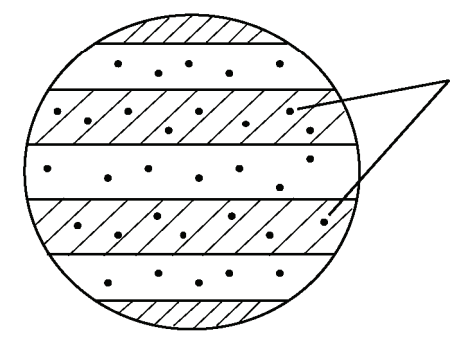

Sputtered Pd-particles

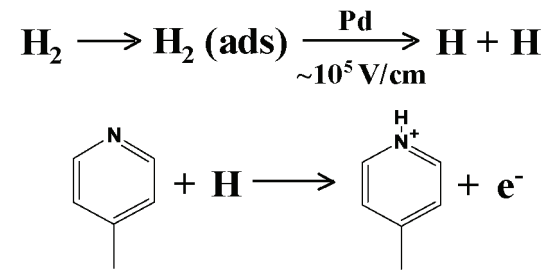




\subsection{Experimental setup for measurements of the Seebeck effect}

In general, carrier determination in highly resistive materials is not an easy task because the number of charge carriers is quite small. Figure 4 shows the experimental setup for measurements of the Seebeck effect for the determination of the charge carrier concentration (Ziman, 1972; Seeger, 1985). This method measures thermoelectric power which appears between hot and cold ends of a material. The hot end is made by a soldering iron with a small spring on top maintained at $100{ }^{\circ} \mathrm{C}$ while the cold end is the ITO electrode at room temperature. If the potential of the hot end is positive, then the carriers are electrons. Similarly, if the potential is negative, the charge carriers are holes.

Since the measurement of the Seebeck effect requires a temperature gradient along the direction of the film thickness, the perylene-imide layer in the $\mathrm{H}_{2}$ gas sensor was prepared thicker (about $1200 \AA$ ) than the standard one (about $300 \AA$ ). Because of this, the present sensor was much less sensitive by about two to three orders of magnitude than the standard one. This trade-off is required for meaningful measurements of the Seebeck effect.

Figure 4 Experimental setup for measurements of Seebeck coefficient

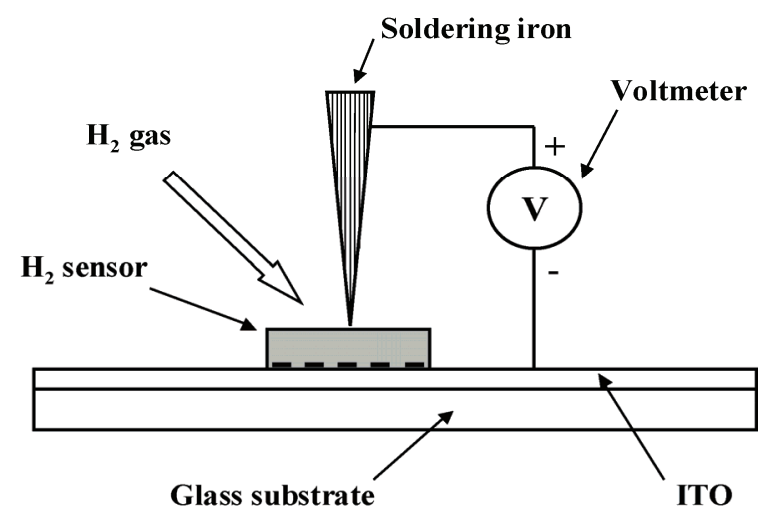

\section{Results and discussion}

\subsection{Crystal structure of $O P P, M P P$ and $P P P$}

Table 1 details the crystallographic parameters for OPP (Mizuguchi et al., 2005), MPP (Mizuguchi et al., 2005) and PPP (Hino et al., 2005).

In the unit cell of OPP, there are two independent molecules, A and B. The ORTEP plot of these molecules are shown in Fig. 5(a). Both molecules A and B are characterized by $C_{\mathrm{i}}$ symmetry. Two pyridyl rings are twisted in the same direction with respect to the perylene-imide skeleton by $77.7^{\circ}$ in molecule A and $72.8^{\circ}$ in molecule B. Molecules A and $\mathrm{B}$ are stacked alternately along the $b$ axis as shown Fig. 5(b). It is apparent that the $\mathrm{N}$ atoms of the pyridyl ring remain unbonded (i.e. free) without being utilized for the formation of, for example, intermolecular hydrogen bonds. Therefore, the $\mathrm{N}$ atoms are in a position to accept protons. This is a prerequisite condition for $\mathrm{H}_{2}$ gas sensors. 
Author

Likewise, the $\mathrm{N}$ atoms of the pyridyl ring in MPP and PPP are found to be free according to the structure analysis. These results are given in Appendix.

Table 1 Crystallographic parameters

\begin{tabular}{cccc}
\hline & $\mathrm{OPP}$ (ortho) & $\mathrm{MPP}$ (meta) & $\mathrm{PPP}($ para $)$ \\
\hline Formula & $\mathrm{C}_{34} \mathrm{H}_{16} \mathrm{~N}_{4} \mathrm{O}_{4}$ & $\mathrm{C}_{34} \mathrm{H}_{16} \mathrm{~N}_{4} \mathrm{O}_{4}$ & $\mathrm{C}_{34} \mathrm{H}_{16} \mathrm{~N}_{4} \mathrm{O}_{4}$ \\
Crystal system & monoclinic & monoclinic & orthorhombic \\
Space group & $P 2_{1} / c$ & $P 2_{1} / n$ & $P c c n$ \\
Molecular symmetry & $C_{\mathrm{i}}$ & $C_{\mathrm{i}}$ & $C_{2}$ \\
$Z$ & 4 & 2 & 4 \\
$a, \AA$ & $17.599(1)$ & $15.422(2)$ & $21.232(2)$ \\
$b, \AA$ & $7.1705(5)$ & $3.8275(6)$ & $15.890(2)$ \\
$c, \AA$ & $20.679(2)$ & $19.282(3)$ & $6.9311(8)$ \\
$\alpha,{ }^{\circ}$ & - & - & - \\
$\beta,{ }^{\circ}$ & $111.004(5)$ & $103.29(1)$ & - \\
$\gamma,{ }^{\circ}$ & - & - & - \\
Density $\left(\mathrm{g} \mathrm{cm}{ }^{-3}\right)$ & 1.49 & 1.63 & 1.55 \\
$R_{1}$ & 0.062 & 0.039 & 0.074 \\
\hline
\end{tabular}

Figure 5 (a) Molecular conformation and (b) molecular arrangement of OPP

(a)

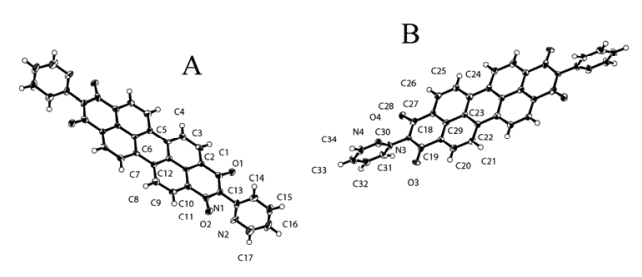

(b)

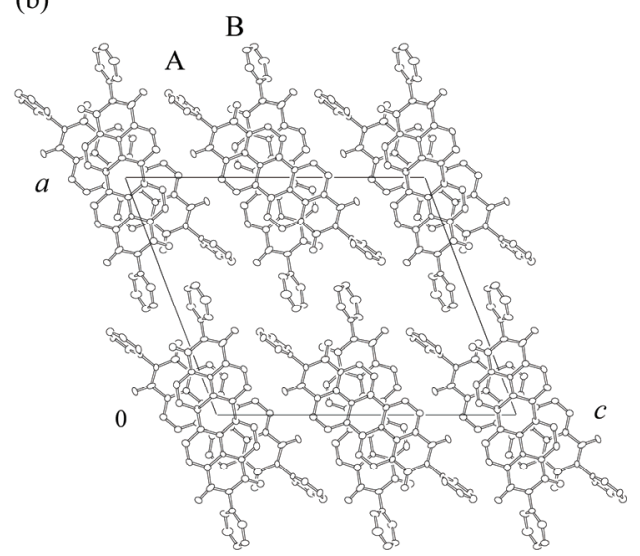

\subsection{Performance of the $\mathrm{H}_{2}$ gas sensor}

Figure 6(a) shows the change in resistivity of the MPP sensor as a function of bias voltage when the sensor is exposed to $100 \% \mathrm{H}_{2}$. It is remarkable to note that the resistivity decreases drastically by four orders of magnitude at room temperature. Likewise, the OPP and PPP sensors show similar sensitivity as shown in Figs. 6(b) and 6(c), respectively. All three sensors exhibit a high sensitivity, but their sensitivity is slightly different, depending on the site of the $\mathrm{N}$ atom.

The concentration dependence of the $\mathrm{H}_{2}$ sensor is shown in Fig. 7 for MPP where the $\mathrm{H}_{2}$ concentrations are $0.01,0.05,0.1,1$ and $100 \%$. Since the initial resistivity is $1.1 \times 10^{8}$ $\Omega \mathrm{cm}$, a reduction of the electrical resistivity by about three orders of magnitude is observed even for the concentration of $0.01 \%$. Furthermore, the resistivity is found to 
Title

decrease linearly with an increase of the $\mathrm{H}_{2}$ concentration. A similar concentration dependence is also observed in the $\mathrm{H}_{2}$ gas sensor based upon OPP and PPP.

Figure 8 shows the build-up of the MPP-based $\mathrm{H}_{2}$ sensor in air when $100 \% \mathrm{H}_{2}$ is on and off, respectively. The build-up time ( $80 \%$ of the maximum time) is $1 \mathrm{~s}$ where the gain is $c a .3 \times 10^{4}$. Gain is defined as the ratio of the initial resistivity to the final one. This indicates that response is quit fast in the gain range of several factors. The signal builds up and then remains nearly constant after a slightly decay. When $\mathrm{H}_{2}$ is switched off, the signal decays and comes back to the initial state in $4 \mathrm{~s}$.

Figure 6 Changes in resistivity of the sensors before and after $\mathrm{H}_{2}(100 \%)$ as a function of applied voltage (a) MPP, (b) OPP and (c) PPP

(a)

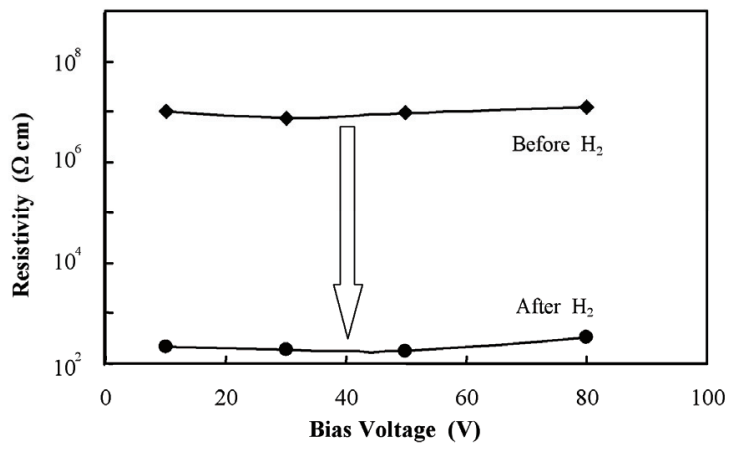

(b)

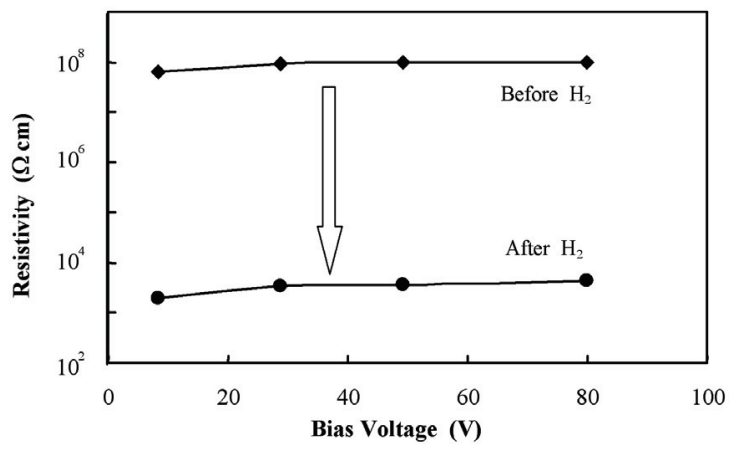

(c)

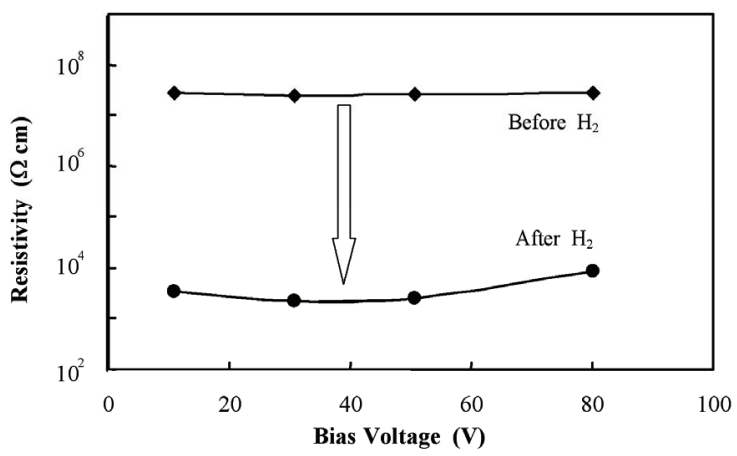


Author

Figure 7 Log-log plot of the resistivity of the MPP sensor and $\mathrm{H}_{2}$ concentration: $0.01,0.05,0.1,1$ and $100 \% \mathrm{H}_{2}$

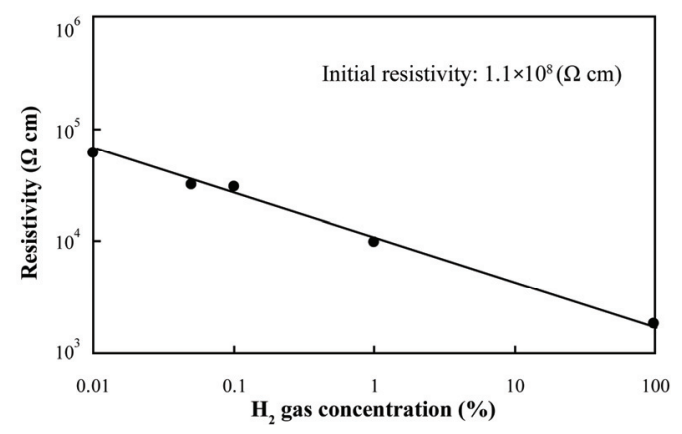

Figure 8 Build-up and build-down of the MPP-based $\mathrm{H}_{2}$ sensor in air when $100 \% \mathrm{H}_{2}$ is on and off, respectively

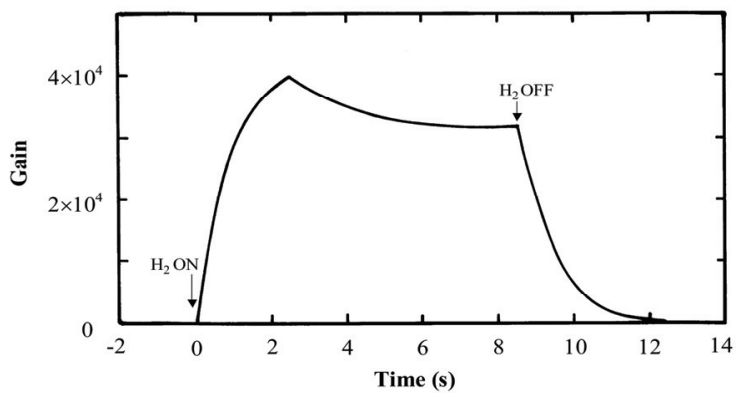

\subsection{Determination of the charge carrier}

Figure 9 shows the thermoelectric power of the PPP sensor as a function of time. A positive potential appears, in the absence of $\mathrm{H}_{2}$, as soon as the contact is made between soldering iron and sample. Then upon introduction of $100 \% \mathrm{H}_{2}$, an additional increase in positive potential is recognized. This clearly indicates that the charge carrier is due to electrons. The present result directly bears out our proposed operation principle shown in Fig. 3(b). Likewise, the OPP and MPP sensors exhibit the same behaviour.

Figure 9 Thermoelectric power of the PPP sensor

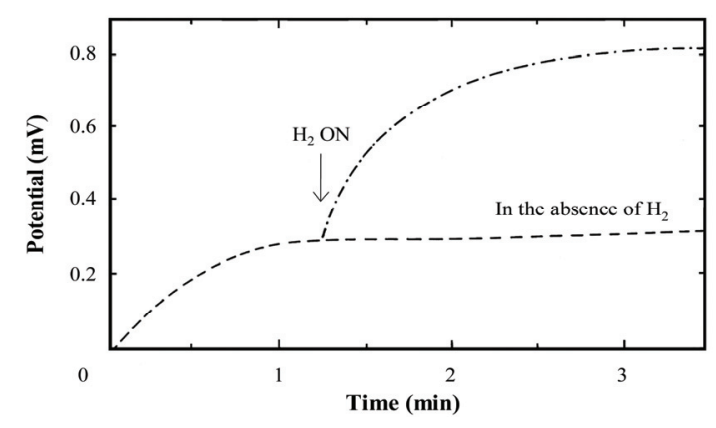


Title

\subsection{Influence of various gases on the gas sensor}

We have studied the influence of various gases on the sensing characteristic: $\mathrm{CH}_{4}(1 \%)$, $\mathrm{CO}(2 \%), \mathrm{CO}_{2}(24 \%), \mathrm{NO}(0.6 \%), \mathrm{SO}_{2}(0.2 \%)$, saturated vapour of $\mathrm{CH}_{3} \mathrm{OH}, \mathrm{C}_{2} \mathrm{H}_{5} \mathrm{OH}$ and $\mathrm{H}_{2} \mathrm{O}$ moisture. The sensor was exposed to these gases with a flow rate of $2 \mathrm{l} / \mathrm{min}$. No noticeable effect (i.e. less than $0.1 \%$ in resistivity change) was recognized for these gases, indicating that the sensors are free from the influence of ambient gases. The present result is understandable as judged from the operation principle described in section 2.2.

\section{Conclusions}

High-performance $\mathrm{H}_{2}$ gas sensors have been developed that utilize the proton affinity of perylene-imide derivatives with pyridyl rings. The two pyridyl rings are found to be unbonded as shown by X-ray analysis and are thus available for protonation necessary for $\mathrm{H}_{2}$ gas sensors. The $\mathrm{H}_{2}$ sensor exhibits a reduction of resistivity by about three orders of magnitude at room temperature even under $0.01 \% \mathrm{H}_{2}$. Furthermore, the process is reversible, and the response is quite fast. The charge carrier is found to be electrons on the basis of the Seebeck effect. No noticeable effect of ambient gases $\left(\mathrm{CH}_{4}, \mathrm{CO}, \mathrm{CO}_{2}\right.$, $\mathrm{NO}, \mathrm{SO}_{2}, \mathrm{CH}_{3} \mathrm{OH}, \mathrm{C}_{2} \mathrm{H}_{5} \mathrm{OH}$, and $\mathrm{H}_{2} \mathrm{O}$ moisture) on the sensors is recognized.

\section{Acknowledgement}

The authors express their sincere thanks to Dr. S. Suzuki for suggestions and discussion.

\section{References}

Duff, J., Hor, A. M., Melnyk, A. R. and Teney, D. (1990) 'Spectral Response and Xerographic Electrical Characteristics of Some Perylene Bisimide Pigments', Proceedings of SPIE, Hard Copy and Printing, Media, and Process, Vol. 1253, pp.183-191.

Herbst, W. and Hunger, K. (1993) Industrial Organic Pigments, Second Edition, VCH, Weinheim, pp.467-475.

Hino, K., Sato, K., Takahashi, H., Suzuki, S. and Mizuguchi, J. (2005) 'N,N'-Di-4-pyridylperylene3,4:9,10-bis(dicarboximide)', Acta Cryst., Vol. E61, pp.o440-o441.

Hirota, T., Imoda, T., Takahashi, H. and Mizuguchi, J. (2006) '3,6-Di-3-pyridylpyrrolo[3,4c]pyrrole-1,4(2H,5H)-dione', Acta Cryst., Vol. E62, pp.o111-o113.

Imoda, T., Hirota, T., Takahashi, H. and Mizuguchi, J. (2005) '3,6-Di-2-pyridylpyrrolo[3,4c]pyrrole-1,4(2H,5H)-dione', Acta Cryst., Vol. E61, pp.o616-o618.

Loutfy, R. O., Hor, A. M., Kazmaier, P. and Tam, M. (1989) 'Layered Organic Photoconductive (OPC) Devices Incorporating $N, N$ '-Disubstituted Diimide and Bisarylimidazole Derivatives of perylene-3,4,9,10-Tetracarboxylic Acid', J. Imag. Sci. Vol. 33, pp.151-159.

Mizuguchi, J. (1981) 'An improved method for purification of $\beta$-copper phthalocyanine', Krist. Tech., Vol. 16, pp.695-700.

Mizuguchi, J. (1993) 'Solution and solid state properties of 1,4-diketo-3,6-bis-(4'-pyridyl)-pyrrolo$[3,4-c]$-pyrrole on protonation and deprotonation', Ber. Bunsenges. Phys. Chem., Vol. 97, pp.684-693.

Mizuguchi, J. (1998) 'Electronic characterization of $N, N$ '-bis(2-phenylethyl)perylene-3,4:9,10bis(dicarboximide) and its application to optical disks', J. Appl. Phys., Vol. 84, pp.4479-4486. 
Author

Mizuguchi, J., Hino, K., Sato, K., Takahashi, H. and Suzuki, S. (2005) 'N,N’-Di-2-pyridylperylene3,4:9,10-bis(dicarboximide)', Acta Cryst., Vol. E61, pp.o437-o439.

Mizuguchi, J., Hino, K., Sato, K., Takahashi, H. and Suzuki, S. (2005) ' $N, N$ '-Di-3-pyridylperylene3,4:9,10-bis(dicarboximide)', Acta Cryst., Vol. E61, pp.o434-o436.

Seeger, K. (1985) Semiconductor Physics, Third Edition, Springer-Verlag, Berlin Heidelberg, New York, Tokyo,

Takahashi, H. and Mizuguchi, J. (2005) 'Hydrogen Gas Sensor Utilizing a High Proton Affinity of Pyrrolopyrrole Derivatives', J. Electrochem. Soc., Vol. 152, pp.H69-H73.

Ziman, J. M. (1972) Principles of the Theory of Solids, Second Edition, Cambridge at the University Press.

\section{Appendix}

The ORTEP plot of MPP is shown in Fig. 10(a). The molecule is characterized by $C_{\mathrm{i}}$ symmetry. Two pyridyl rings are twisted in the same directions by $54.9^{\circ}$. The molecules are stacked in a fashion 'hunter's fence' along the $b$ axis, as shown in Fig. 10(b). The N atoms of the pyridyl ring are found to be unbonded.

Figure 11(a) shows the ORTEP plot of PPP. Contrary to OPP and MPP molecules, the PPP molecule has $C_{2}$ symmetry. Therefore, two pyridyl rings are twisted in opposite directions by $74.5^{\circ}$. The molecules are stacked along the $c$ axis with a tilt angle of $31.5^{\circ}$ between adjacent molecules as shown in Fig. 11(b). Here again, the $\mathrm{N}$ atoms of the pyridyl ring are free and thus in a position to accept protons.

Figure 10 (a) Molecular conformation and (b) molecular arrangement of MPP

(a)

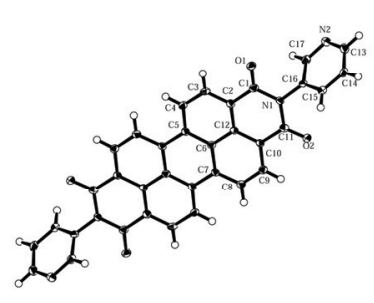

(b)

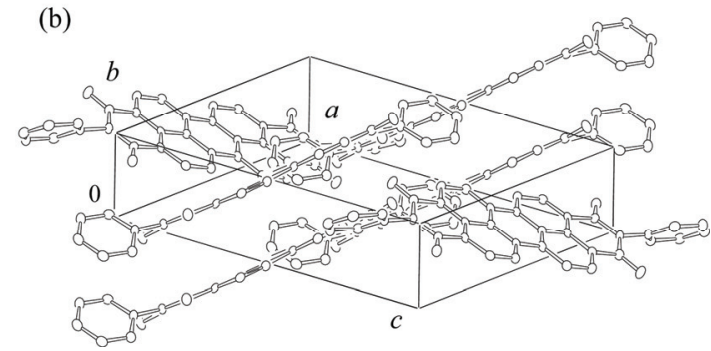

Figure 11 (a) Molecular conformation and (b) molecular arrangement of PPP

(a)

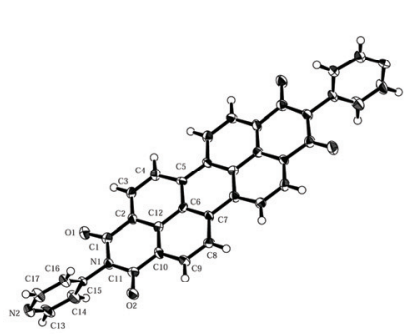

(b)

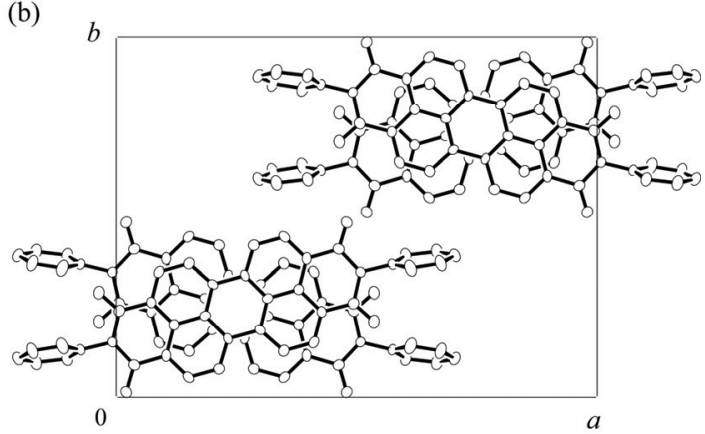

